\title{
Optimal Setup of the DLR MiroSurge Telerobotic System for Minimally Invasive Surgery
}

\author{
Rainer Konietschke, Tim Bodenmüller, Christian Rink, Andrea Schwier, Berthold Bäuml, and Gerd Hirzinger
}

\begin{abstract}
This video presents the complete procedure for the optimal setup of the DLR MiroSurge telerobotic system for minimally invasive surgery. Two key features are implemented. First, optimization algorithms preoperatively determine several setups that are then rated and selected by the surgeon. Second, the intraoperative situation is taken into account. The newly developed VR-Map device together with fast registration and optimization algorithms enable a quick procedure to assure the optimal patient-specific setup of the robotic system.
\end{abstract}

\section{INTRODUCTION}

The use of robotic assistance in minimally invasive surgical procedures may increase the overall task complexity, setup time, and the potential number of error sources. We present here a patient-specific setup procedure for the DLR MiroSurge Telerobotic System for Minimally Invasive Surgery [1] that addresses all these issues. The procedure (see Fig. 1) covers all steps, from the preoperative planning to the intra-operative setup of the system.

The next Section describes the preoperative phase, and Section III presents the method for fast intraoperative setup, congruent with the plan. Section IV closes the paper with a summary and outlook.

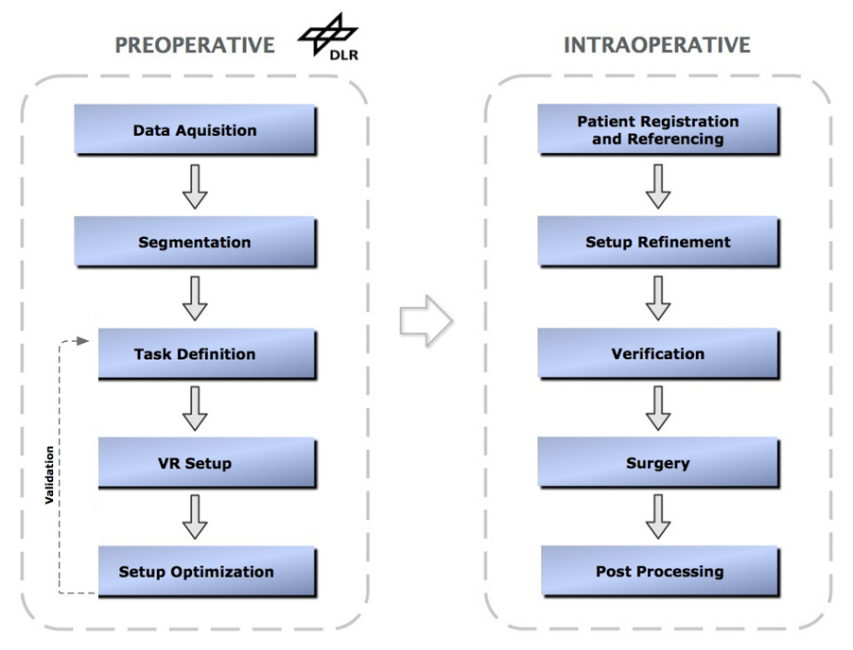

Fig. 1. Procedure for preoperative planning and the intra-operative setup for the MiroSurge system.

\section{Preoperative Planning}

Preoperatively, computer tomography (CT) data of the patient is segmented to get 3D surfaces of all relevant

The authors are affiliated with the Institute of Robotics and Mechatronics, German Aerospace Center (DLR), Wessling, Germany, rainer.konietschke@dlr.de structures. Then, the surgeon outlines the desired operation area in the simulation and generates a $3 \mathrm{D}$ volume. On the 3D surface of the patient skin, proposed locations for the instrument ports are marked. The surgeon also selects the number of robots and their coarse position in space for the simulation.

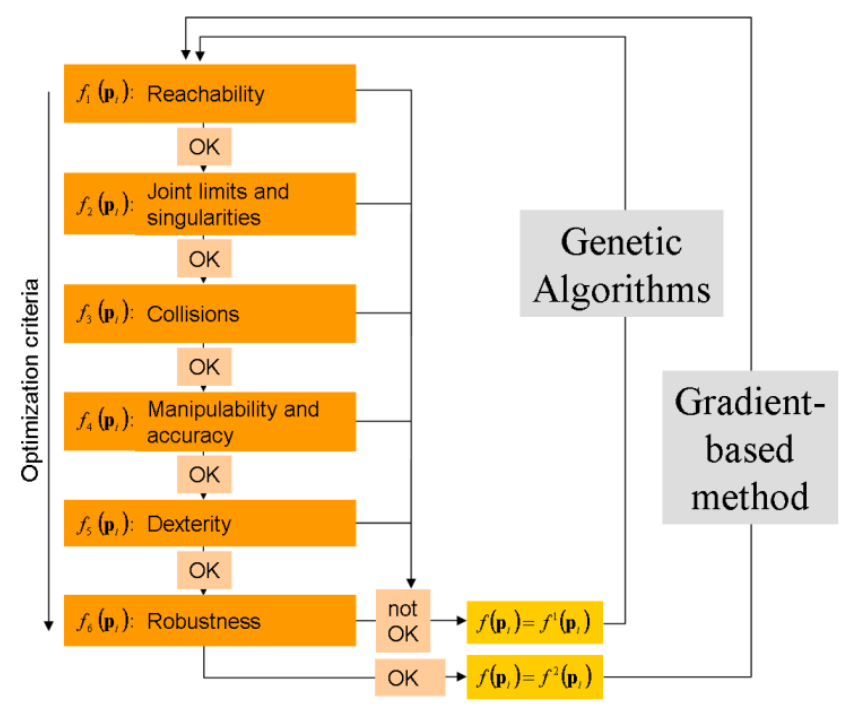

Fig. 2. Two-stage optimization. Genetic algorithms are used to optimize the non-continuous function $f^{1}$ and to generate solutions with high variability in terms of the optimization parameters $\mathbf{p}$. In the second stage, the continuous function $f^{2}$ is optimized with a gradient based method to find the local minima for the ten best solutions.

Algorithms then calculate candidates of optimal setups with respect to various criteria (see Fig. 2 and [2]). First, configurations that satisfy a certain minimal quality for all criteria are chosen. Genetic Algorithms generate solutions with high variability. Then the setups are locally optimized using a gradient based method. The computation time of the optimization mainly depends on the size of the search space. For the problem shown in the video, solutions are found within five minutes on a standard PC. As result of the optimization, the ten best solutions are presented to the experienced surgeon to choose between. This completes the preoperative planning.

\section{INTRAOPERATIVE SETUP}

In the operating room, the setup has to be adjusted to the real situation. Rapid completion of this step is important in order to reduce idle times of the operating room. The VRMap device was newly designed to assist the clinician during 
this phase [3]. This device scans 3D surfaces and projects data onto them. The implementation of the VR-Map system integrates two AVT Guppy F-046C Firewire cameras with Pentax lenses $(f=6 \mathrm{~mm})$, a Scanlab ScanCube 7 laser projector, and a laser-line module with $650 \mathrm{~nm}$ wavelength, $5 \mathrm{~mW}$ laser power, and $60^{\circ}$ opening angle. The weight of the system is $1,3 \mathrm{~kg}$. Its dimension is $242.5 \mathrm{~mm} \mathrm{x}$ $100 \mathrm{~mm} \times 140 \mathrm{~mm}$. The mechanical concept of the system is shown in Fig. 3. The cameras are tilted by $10^{\circ}$ and have a base distance of $55 \mathrm{~mm}$, which is a trade-off between perspective difference and range precisions. The laserline module is separately inclinable $\left(0^{\circ}-10^{\circ}\right)$ for different measurement ranges. At a laser-beam tilt angle of $5^{\circ}$, this results in a minimum scan distance of about $138 \mathrm{~mm}$. The main component of the AutoPointer is a ScanCube 7 laser projector. Its position-controlled high speed gyro-scanners deflect a laser beam in two axes. Hence, it can project complex cutting trajectories and multiple symbols at the same time. A mechanical interface for the DLR medical robot MIRO magnetic mount is attached on the rear side of the VR-Map.

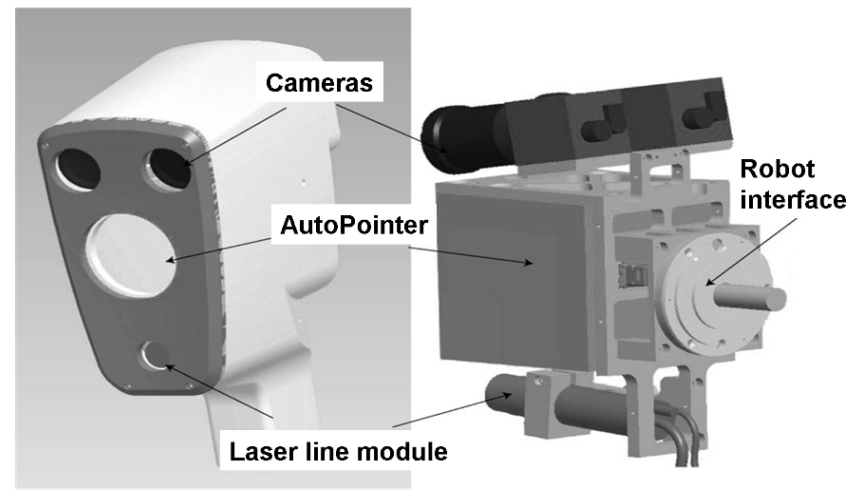

Fig. 3. Mechanical concept and components of the VR-Map with and without casing.

The whole workflow is controlled with a handheld device. First, the patient's body is scanned. Therefore, the VRMap is moved by touching the robot (see Fig. 4). The preoperative patient data is then registered with the scanned 3D surface. The robot base positions are restricted along the table. Therefore, it is not possible to simply shift the planned setup to fit with the real patient. Instead, the setup is re-optimized using a fast gradient based search. Then, the optimized positions for the robot bases are projected onto the table with the projector-function of the VR-Map. The robots are adjusted accordingly. In a last step, the optimal entry points are projected onto the patient's body (see Fig. 5), and the entry points for the endoscopic instruments are set.

This assures an optimal setup for the operation.

\section{Summary}

The video shows a complete setup procedure for the MiroSurge system with two key features. Preoperatively, optimization algorithms determine several setups that are then rated and selected by the surgeon. Intraoperatively, the

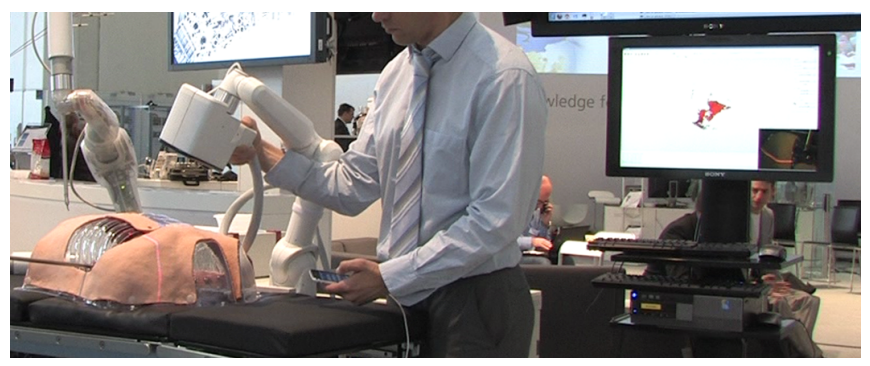

Fig. 4. The VR-Map is mounted to one of the medical robots and guided by hand to scan the patient dummy.

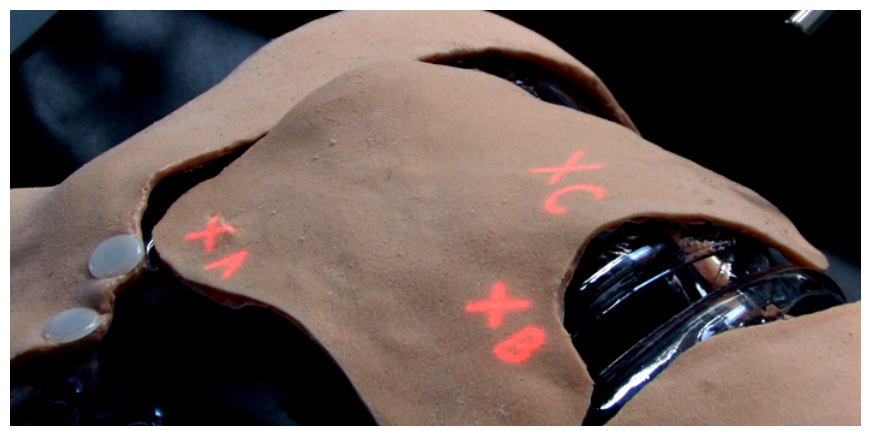

Fig. 5. The optimized entry point locations are projected onto the patient dummy.

actual situation is taken into account. The VR-Map device together with fast registration and optimization algorithms enable a quick and accurate procedure to assure the optimal patient-specific setup of the robotic system.

In future work, the phase subsequent to the surgical intervention will be investigated. Comparisons between the preoperative plan and the actual setup in the operating room may help to speed up optimization and to increase robustness of the procedure. Generation of templates will assist the generation of new patient specific plans. Furthermore, the versatility of the planning tools will be tested in new application areas, e.g. in robotic assistance in open surgery.

\section{REFERENCES}

[1] U. Hagn, R. Konietschke, A. Tobergte, M. Nickl, S. Jörg, B. Kübler, G. Passig, M. Gröger, F. Fröhlich, U. Seibold, L. Le-Tien, A. AlbuSchäffer, A. Nothhelfer, F. Hacker, M. Grebenstein, and G. Hirzinger. DLR MiroSurge: a Versatile System for Research in Endoscopic Telesurgery. International Journal of Computer Assisted Radiology and Surgery, 5(2):183-93, 2010.

[2] Rainer Konietschke. Planning of Workplaces with Multiple Kinematically Redundant Robots. Technische Universität München, Munich, Germany, 2007. PhD Thesis.

[3] A. Schwier, R. Konietschke, T. Bodenmüller, T. Ende, S. Kielhöfer, and G. Hirzinger. VR-Map: A New Device for Patient Registration and Optimal Robot Positioning. In 9. Jahrestagung der Deutschen Gesellschaft für Computer- und Roboterassistierte Chirurgie (CURAC), Düsseldorf, Germany, 18.-19. November 2010. 